\title{
Propiedades neuroprotectoras de los esteroides sexuales y los neuroesteroides
}

\author{
S. Veiga ${ }^{a}$, L.M. García-Segura ${ }^{\text {a }}$, I. Azcoitia ${ }^{\text {b }}$
}

\section{THE NEUROPROTECTIVE PROPERTIES OF SEX STEROIDS AND NEUROSTEROIDS}

\begin{abstract}
Summary. Introduction. The nervous system is a target for steroid hormones as well as a steroidogenic tissue, and it produces steroids that have a paracrine or autocrine effect on neurons and glial cells. Steroids formed in nervous tissue are called neurosteroids in order to differentiate them, in terms of their origin, from the peripheral steroids, although they both share the same molecular structure. Development. We analyse the capacity of neurons and glial cells to synthesise steroids and describe the role played in steroidogenesis by certain key molecules, such as steroidogenic acute regulatory protein, peripheral benzodiazepine receptor and aromatase enzyme, which acts as a catalyst in the conversion of testosterone into estradiol. We also provide a description of the different mechanisms of action of the hormonal steroids and neurosteroids in the nervous system. These include both the regulation of protein synthesis by neurons and glial cells, by acting on nuclear receptors, and rapid effects mediated by membrane receptors or the allosteric modulation of neurotransmitter receptors. We review the clinical and experimental evidence for the neuroprotective effects of sex steroids and neurosteroids, and the limitations of hormone replacement therapy following menopause. Conclusions. Given the restraints involved in the systemic use of hormones as neuroprotective therapy, alternative strategies that take advantage of the neuroprotective properties of steroids must be sought. These could involve locally increasing their synthesis inside the brain or developing molecules that activate the steroid receptors in the nervous system and not in the peripheral organs. [REV NEUROL 2004; 39: 1043-51]
\end{abstract}

Key words. Aromatase. Dehydroepiandrosterone. Estradiol. Neuroprotection. Neurosteroids. Peripheral benzodiazepine receptor. Pregnenolone. Progesterone. Steroidogenesis. Steroidogenic acute regulatory protein. Testosterone.

\section{INTRODUCCIÓN}

El cuerpo y el cerebro se comunican entre sí, no sólo a través del sistema nervioso periférico (SNP), sino también a través de la sangre, mediante mensajes hormonales. De hecho, podemos considerar al cerebro como el órgano endocrino más importante del cuerpo, si nos atenemos al número de hormonas que se sintetizan en el hipotálamo. Por otra parte, la actividad neural se afecta por las secreciones humorales de las glándulas endocrinas periféricas. Una de las clases de hormonas que más afectan al desarrollo y la función cerebral son las hormonas sexuales: andrógenos, estrógenos y progestágenos. Estas hormonas, esteroides derivados del colesterol, actúan sobre las neuronas, las sinapsis y las células gliales, y regulan la supervivencia, diferenciación y conectividad de grupos neuronales específicos, tanto en el cerebro, como en la médula espinal. Muchos de los efectos de las hormonas sexuales durante el desarrollo del sistema nervioso (SN) tienen carácter permanente y repercuten en fenómenos de gran importancia, como la diferenciación sexual del cerebro. También en la etapa adulta las hormonas sexuales influyen en la función nerviosa, y regulan la transmisión sináptica, la síntesis de neurotransmisores, la expresión de receptores para transmisores, y la plasticidad de las conexiones neuronales.

Durante muchos años se consideró que las acciones de las hormonas sexuales en el cerebro se circunscribían principalmente a las regiones involucradas en la regulación neuroendocrina y el control del comportamiento. Hoy se ha establecido bien que las hormonas sexuales ejercen un amplio espectro de acciones

Recibido: 02.03.04. Aceptado tras revisión externa sin modificaciones: 02.04.04.

${ }^{a}$ Instituto Cajal. CSIC. ${ }^{b}$ Departamento de Biología Celular. Facultad de Biología. Universidad Complutense. Madrid, España.

Correspondencia: Dr. Luis M. García Segura. Instituto Cajal. CSIC. Avda. Dr. Arce, 37.E-28002 Madrid.E-mail:Imgs@cajal.csic.es

(C) 2004, REVISTA DE NEUROLOGÍA tróficas en muchas otras áreas cerebrales. Además, cada vez resulta más claro que el cerebro es capaz de sintetizar estas mismas moléculas, que actuarán localmente como neuromoduladores y a las que ya no podemos llamar propiamente hormonas, ya que van a tener un modo de acción paracrino o autocrino. Por otra parte, existe una amplia evidencia experimental que indica que los esteroides sexuales tienen efectos neuroprotectores. En este trabajo se revisan las evidencias que indican que el SN tiene la capacidad de sintetizar esteroides, se analizan los diversos mecanismos de acción de estas moléculas y las evidencias clínicas y experimentales sobre sus efectos neuroprotectores. Finalmente, se sugieren posibles estrategias neuroprotectoras basadas en la formación local de esteroides por el SN.

\section{SÍNTESIS DE ESTEROIDES EN EL SISTEMA NERVIOSO}

El cerebro es capaz de sintetizar esteroides a partir del colesterol y de metabolizar los esteroides hormonales en derivados que pueden ser potentes neuromoduladores. Por ejemplo, el cerebro sintetiza progesterona y metaboliza esta molécula para formar sus derivados reducidos, dihidroprogesterona (DHP) y tetrahidroprogesterona (THP). La THP modula la actividad de los receptores $\mathrm{GABA}_{\mathrm{A}}$ y es un potente ansiolítico, con efectos antidepresivos $[1,2]$. Esta molécula aumenta la eficacia antidepresiva del tratamiento con inhibidores de la recaptación de serotonina. Además, el tratamiento con estos inhibidores aumenta los niveles de THP en el cerebro [3,4]. Todo esto sugiere que puede existir una interferencia entre las acciones de las hormonas sexuales en el cerebro con las acciones de esas mismas moléculas sintetizadas localmente. Por ejemplo, durante el embarazo el cerebro se expone a altos niveles de progesterona hormonal y, por lo tanto, dispone de elevadas cantidades de precursor para sintetizar THP. El abrupto descenso de los niveles de progestero- 
na hormonal tras el parto priva al cerebro de esta fuente de THP. Esto puede favorecer el desarrollo de las depresiones posparto, quizá porque el cerebro de muchas mujeres tarda en readaptar su producción local de progesterona para producir THP. Situaciones similares se pueden dar a consecuencia del cese de la producción de hormonas por el ovario en la menopausia y en los varones con el envejecimiento, debido a la disminución de la secreción hormonal por el testículo.

La existencia de una síntesis local de esteroides por el cerebro ha llevado a acuñar el término neuroesteroide y a hacer la distinción entre neuroesteroides y esteroides neuroactivos. Se denomina neuroesteroide a aquel esteroide que se sintetiza en el SN a partir del colesterol y que actúa localmente. Un esteroide neuroactivo es aquel esteroide, ya sea hormona o neuroesteroide, que actúa sobre el SN. La primera evidencia de que los esteroides podían sintetizarse de novo en el cerebro a partir del colesterol surgió en el laboratorio de Baulieu, en donde observaron que ciertos esteroides y sus derivados, tales como la dehidroepiandrosterona (DHEA), la pregnenolona y sus formas sulfatadas, se encontraban en una concentración mayor en el tejido nervioso que en el plasma y que la concentración en el cerebro de dichos esteroides permanecía inalterada tras la extirpación de los principales órganos esteroidogénicos: las gónadas y la médula adrenal $[5,6]$. Estos hechos sugerían la capacidad del SN de producir de novo moléculas de naturaleza esteroídica, que recibieron el calificativo de neuroesteroides, para diferenciarlos de aquellos esteroides de origen no nervioso. En estudios posteriores de varios laboratorios se ha puesto de manifiesto la presencia de enzimas esteroidogénicas funcionales en el $\mathrm{SN}$, a través de medidas de actividad enzimática [7], de la expresión del ARNm [8,9] o de la proteína [10,11]. Estas enzimas son capaces de sintetizar esteroides a partir del colesterol y de metabolizar los esteroides periféricos que alcanzan el SN por el torrente sanguíneo, transformando, por ejemplo, la testosterona en estradiol o la progesterona en THP. La figura 1 recoge un esquema de las principales rutas de síntesis de esteroides en el SN y de las enzimas que participan en ellas.

La presencia de enzimas sintéticas o modificadoras de esteroides es lo que hace del tejido nervioso un tejido esteroidogénico. Se acepta que las principales células esteroidogénicas en el SN son las células gliales [12,13]. En el caso del SNP, está claro que las células de Schwann representan la fuente principal de neuroesteroides. Sin embargo, en el sistema nervioso central (SNC), aparte de los oligodendrocitos y los astrocitos, las neuronas también presentan capacidad esteroidogénica. De hecho, las neuronas y las células gliales presentan diferentes enzimas [12]. Así, la P450scc, responsable de la formación de pregnenolona a partir de colesterol y la $3 \beta-H S D$, responsable de la producción de progesterona y androstenediona, se expresan en neuronas, oligodendrocitos y astrocitos. Por su parte, la $P 450 \mathrm{cl}$, que participa en la formación de DHEA y la P450aro o aroma- tasa, que cataliza la conversión de testosterona en estradiol, únicamente se han encontrado en astrocitos y neuronas, pero no en oligodendrocitos. Sin embargo, la actividad de las enzimas no es igual en los tres tipos celulares; tanto astrocitos, como neuronas y oligodendrocitos son capaces de producir pregnenolona, pero la actividad de $P 450$ scc en oligodendrocitos es mayor que en astrocitos y neuronas. De igual manera, P450aro se encuentra tanto en astrocitos como neuronas, pero en condiciones normales son estas últimas las que presentan una mayor capacidad de aromatización de la testosterona para producir estradiol. La figura 2 resume esta distribución enzimática en el SNC. La capacidad diferencial en la esteroidogénesis cerebral sugiere una contribución tripartita de los tres tipos celulares, que proveería de neuroesteroides al tejido nervioso durante determinados procesos, como el desarrollo cerebral o en estados carenciales, como la menopausia.

La etapa limitante de la ruta de esteroidogénesis es el transporte del precursor colesterol a la membrana mitocondrial interna donde se encuentra localizada la primera enzima esteroidogénica (P450 scc), que transforma el colesterol en pregnenolona. Todavía no se conoce por completo el proceso de transporte de colesterol de la membrana mitocondrial externa, muy rica en colesterol, a la membrana mitocondrial interna, pobre en colesterol, a través de un espacio intermembranal acuoso. Por lo menos, hay dos proteínas implicadas en su transporte a través de la membrana mitocondrial: la proteína de regulación aguda de esteroidogénesis, StAR (del inglés, steroidogenic acute regulatory protein) y el receptor periférico de benzodiacepinas, PBR (del inglés, peripheral-type benzodiazepine receptor). Ambas proteínas se localizan en la membrana mitocondrial externa, en los puntos de contacto con la interna. Aunque no se ha demostrado una interacción directa entre StAR y PBR, sí se sabe que se encuentran físicamente muy cercanas [14] y que, por tanto, la colaboración es muy plausible. Así, se ha propuesto que StAR capta el colesterol de la 


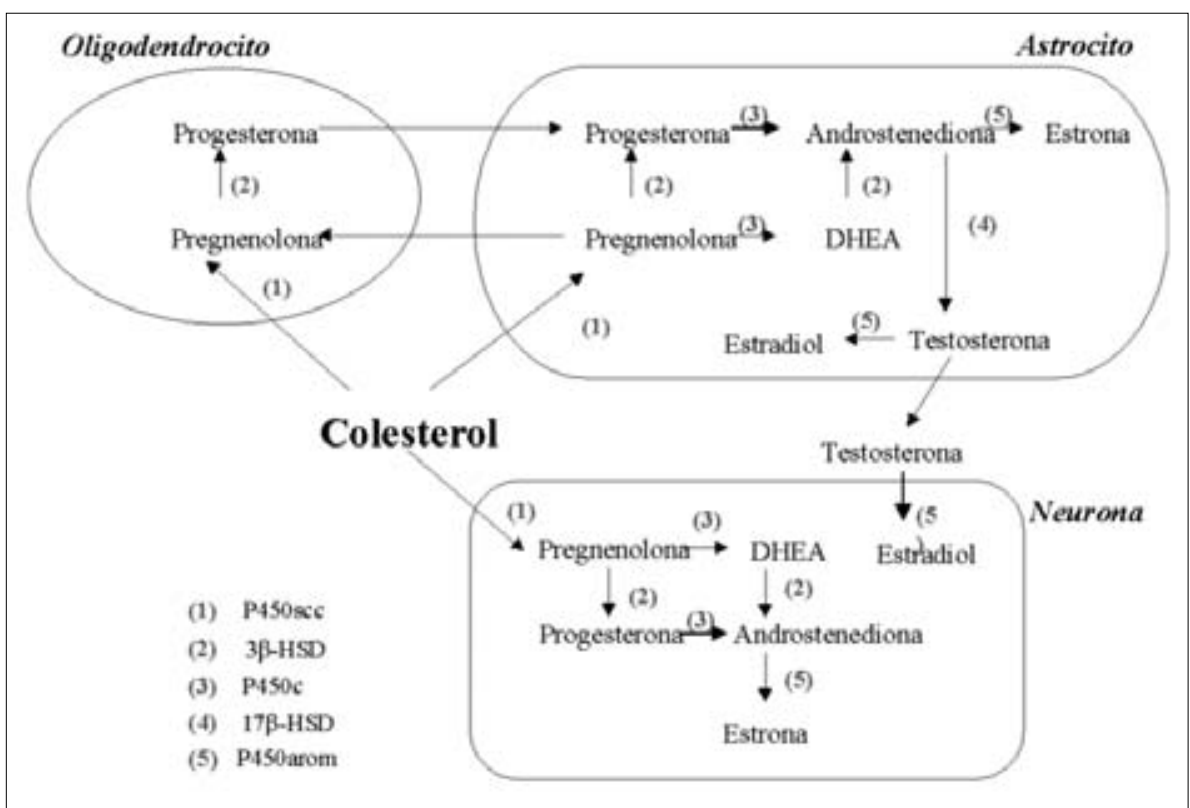

Figura 2. Participación de astrocitos, oligodendrocitos y neuronas en la síntesis de esteroides en cerebro de rata. Basado en [12].

membrana mitocondrial externa y lo transfiere a PBR, que formaría el canal de colesterol dirigiéndolo a la membrana mitocondrial interna para usarse como sustrato de P450scc [15].

La regulación de StAR es aguda, muy rápida y fundamentalmente transcripcional. StAR se expresa ampliamente en el SNC. $\mathrm{Su}$ expresión es principalmente neuronal, pero también se encuentra en astrocitos [16]. En el SNP, StAR se expresa en las células de Schwann [17]. Recientemente se ha demostrado que la expresión de StAR en neuronas aumenta tras lesión cerebral y durante el envejecimiento [16]. Esto sugiere que el cerebro puede modificar su tasa de esteroidogénesis en estas condiciones.

El PBR difiere de los receptores centrales de benzodiacepinas, entre otras propiedades, por su falta de acoplamiento a los receptores de $\mathrm{GABA}_{\mathrm{A}}$ y por su localización en la membrana mitocondrial. En el SN adulto, los niveles de PBR son muy bajos. Sin embargo, esta proteína aumenta notablemente su expresión en las células de glía, tras sección del nervio ciático $[18,19]$, en encefalitis causadas por herpes [20], en encefalomielitis autoinmune experimental [21], en pacientes con esclerosis múltiple [22], en isquemia [23], tras una lesión traumática [24] y tras inyecciones de neurotóxicos [25-27]. El aumento de PBR en el SNC se produce mayoritariamente en la microglia. Tanto es así que un ligando de PBR, el PK11195, se utiliza como marcador de microglia activada [23,28-30]. El aumento en la expresión de PBR tras lesión, junto con el aumento en la expresión de StAR en las mismas condiciones, sugiere que el cerebro aumenta localmente la síntesis de esteroides como un mecanismo de protección. Existen agonistas y antagonistas de PBR disponibles y la industria desarrolla nuevas moléculas con estas propiedades. Una importante aplicación de los antagonistas de PBR es el tratamiento del cáncer, ya que PBR regula la apoptosis $[31,32]$. Otra importante aplicación de los moduladores del PBR deriva de su papel en situaciones de ansiedad y depresión, probablemente mediado por la síntesis local de esteroides con propiedades ansiolíticas [33-35]. La posibilidad de que ligandos de PBR tengan efectos neuroprotectores se encuentra actualmente en estudio.

\section{MECANISMOS DE ACCIÓN DE LOS ESTEROIDES EN EL SISTEMA NERVIOSO}

El mecanismo clásico de acción de las hormonas sexuales en el SN es a través de la interacción con sus receptores intracelulares: los receptores de estrógeno $\alpha$ y, el receptor de andrógenos y el receptor de progesterona. Estos receptores son factores nucleares de trascripción, es decir, proteínas que se unen al ADN y regulan la trascripción de genes específicos. De esta manera, las hormonas sexuales aumentan o disminuyen la síntesis de determinadas proteínas.

Además de los receptores nucleares, la existencia de receptores para esteroides en la membrana celular se ha documentado bien [36,37]. Se han descrito sitios de unión a membrana para varios esteroides, que incluyen la progesterona [38], vitamina D [39], testosterona [40], DHEA [41] y estrógenos [42]. Los efectos que desencadenan han dado en llamarse 'no transcripcionales', ya que la unión del esteroide con su receptor no provoca una modificación directa en la expresión génica, sino que supone una respuesta celular más rápida y que parece involucrar la intervención de otros receptores de membrana y/o vías de señalización intracelular. No obstante, no debe descartarse un efecto a largo plazo, ya que se modifican parámetros celulares como la concentración intracelular de $\mathrm{Ca}^{2+} \mathrm{o}$ la regulación de cinasas, que en último término pueden variar la expresión de determinados genes. Por tanto, los efectos de un esteroide son de amplio intervalo y abarcan efectos genómicos y no genómicos, que muchas veces pueden ser mecanismos complementarios en la respuesta celular a un esteroide.

Otro mecanismo de acción de los esteroides muy importante en el SN es la modulación alostérica de receptores ionotrópicos y de transportadores de neurotransmisores [43]. La modulación alostérica, al igual que la interacción del esteroide con receptores de membrana específicos, genera inicialmente respuestas independientes de la síntesis de ARN o de proteínas, por lo que sus efectos entrarían también en la categoría de 'no transcripcionales'. Uno de los primeros efectos rápidos observados de los esteroides en el SN fue la regulación de corrientes iónicas a través de la membrana celular. Este fenómeno se determina por la interacción de los esteroides con receptores para neurotransmisores. Casi la totalidad de los receptores ionotrópicos conocidos modifican su función por los esteroides $[43,44]$. Este es el caso de la modulación alostérica de los receptores $\mathrm{GABA}_{\mathrm{A}}$ [45]. Por ejemplo, la THP es un modulador positivo de los receptores $\mathrm{GABA}_{\mathrm{A}}$, ya que aumenta el flujo de $\mathrm{Cl}^{-}$al producir un incremento en la frecuencia y/o duración de apertura de dicho canal iónico. También se ha descrito la modulación alostérica por esteroides de otros receptores para neurotransmisores ionotrópicos, como son los de serotonina 5-HT 3 [46,47], NMDA [48,49], AMPA [50], kaínico [50], glicina [51] y los nicotínicos de acetilcolina [52,53]. La modulación alostérica de los receptores para neurotransmisores genera respuestas que van desde milisegundos a segundos, por lo que éste constituye un mecanismo de acción muy rápido de los esteroides. 
Los esteroides también pueden ejercer sus efectos en las células nerviosas sin interaccionar con receptores. Por ejemplo, algunos esteroides poseen efectos antioxidantes que son independientes de receptores. La presencia de un grupo hidroxilo en posición 3 del anillo A o de un anillo bencénico con un átomo de hidrógeno libre permite neutralizar a moléculas con electrones desapareados, como es el caso de los radicales libres. Los radicales libres son moléculas altamente reactivas que provocan daños en el ADN y en las membranas celulares, y que pueden producir la muerte celular si se desbordan las defensas antioxidantes de la célula [54]. El estradiol posee ambas características estructurales, por lo que no es sorprendente que sea el esteroide más estudiado al respecto y cuyas capacidades antioxidantes se describen mejor [55]. Sin embargo, otros esteroides como la DHEA, la pregnenolona o la THP presentan un grupo hidroxilo en la posición 3 del anillo A y son, por tanto, antioxidantes potenciales. De hecho, se ha

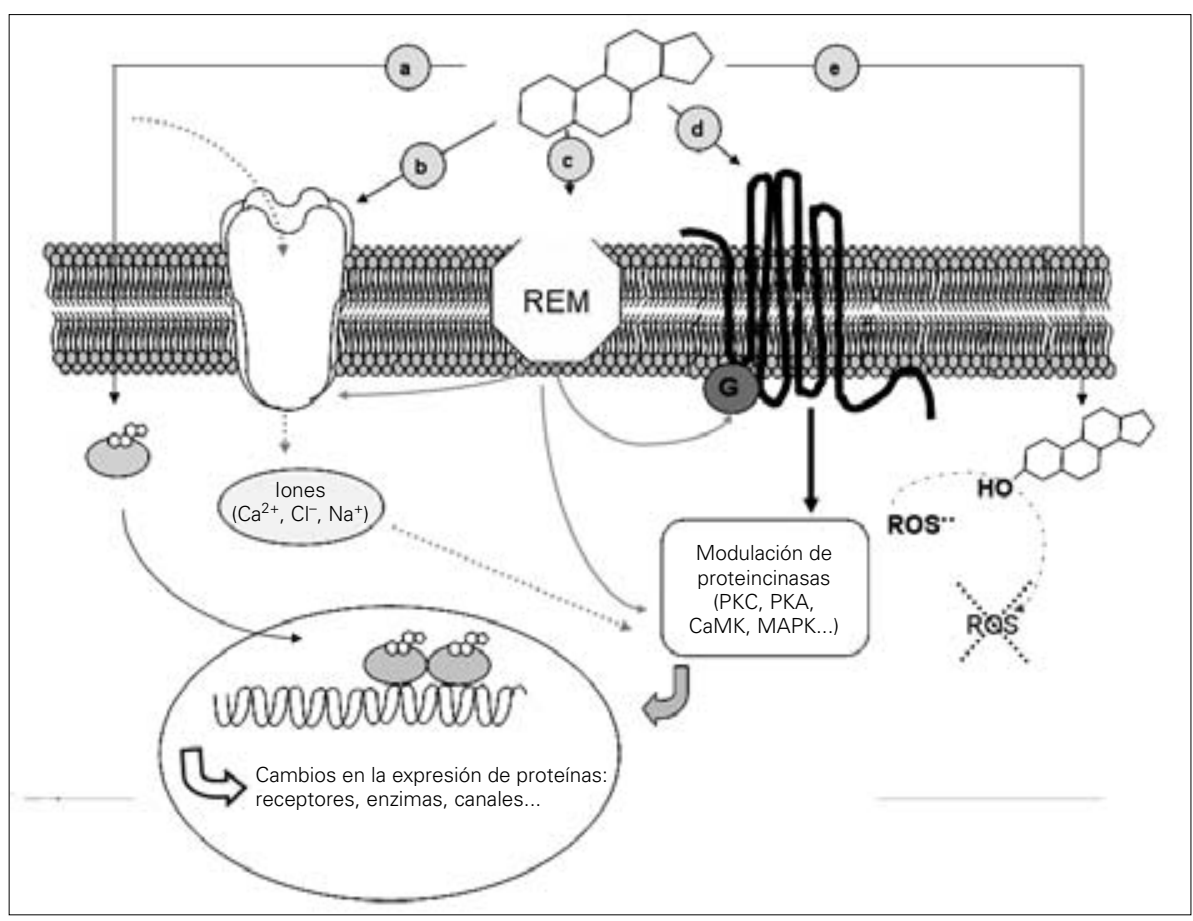

Figura 3. Mecanismos de acción de los esteroides en el sistema nervioso. (a): mecanismo clásico: interacción con receptores intracelulares; (b): modulación alostérica de canales iónicos de membrana; (c): interacción con el receptor putativo de membrana; (d): modulación de la actividad de receptores acoplados a proteínas G; (e): secuestro de radicales libres. REM: receptor de esteroides de membrana; ROS: compuestos reactivos de oxígeno. demostrado la capacidad antioxidante de la DHEA en varios modelos experimentales de estrés oxidativo, como la privación de oxígeno [56], la exposición a radicales libres [57] o a altas concentraciones de glucosa en cultivo [58].

El efecto antioxidante del estradiol y de la DHEA se ha descrito principalmente in vitro. Las concentraciones que se han usado son elevadas y muy superiores a las concentraciones de estos esteroides en sangre. El requerimiento de dosis suprafisiológicas cuestiona que este mecanismo participe en la neuroprotección observada in vivo. Sin embargo, no se conoce la concentración que puede alcanzarse en una determinada área cerebral como consecuencia de la síntesis local de esteroides. Recientemente se ha puesto de manifiesto que los astrocitos responden a una lesión en el cerebro aumentando la expresión de aromatasa [59], por lo que es posible que se alcancen altas concentraciones de estradiol en la zona de la lesión y que el estradiol pueda actuar como antioxidante.

En resumen, existen varios mecanismos a través de los cuales los esteroides modifican la función nerviosa y sus efectos pueden, en algunos casos, implicar a varias vías simultáneamente. La figura 3 muestra un esquema de estas vías y la posible interacción entre las mismas. Además, la amplia distribución de las enzimas esteroidogénicas en el $\mathrm{SN}$, unida a la capacidad de los esteroides de actuar sobre multitud de dianas, tanto en glía como en neuronas, revela a los esteroides como un factor de vital importancia en la regulación de la función neural.

\section{ESTEROIDES Y DISFUNCIONES DEL SISTEMA NERVIOSO: IMPLICACIONES TERAPÉUTICAS}

Como hemos visto, los esteroides desempeñan un papel importante en multitud de procesos neurales, por lo que no es raro pensar que la alteración en sus niveles pueda implicarse en la etiología de algunas enfermedades neurológicas o psiquiátricas. Uno de los datos que mejor apoyan esta afirmación es la existencia de diferencias sexuales en la recuperación ante daños del tejido nervioso debidos a traumatismos o accidentes cerebrovasculares. Estas diferencias se atribuyeron en un principio a diferencias sexuales en la conectividad y tamaño de los centros nerviosos. Sin embargo, parece ser más específico la presencia de determinados esteroides como el estradiol o la progesterona, en el sitio y en el momento del daño cerebral [60]. Este es un factor de considerable importancia, especialmente en las mujeres, en las que la fase del ciclo menstrual puede influir enormemente en la respuesta del tejido cerebral al daño. De hecho, este fenómeno se ha descrito en ratas, donde la vulnerabilidad de las neuronas del hilus del hipocampo a estímulos neurodegenerativos, varía en función de la fase del ciclo estral [61]. Es más, algunos estudios clínicos han confirmado que las variaciones en los niveles plasmáticos de hormonas sexuales influyen en el riesgo a padecer un infarto cerebral; de esta manera, las mujeres premenopaúsicas presentan una menor incidencia de infarto cerebral que los hombres de la misma edad. Esta protección se pierde cuando las mujeres entran en la menopausia y el riesgo de padecer un infarto cerebral se iguala al de los hombres [62]. Asimismo, existen estudios clínicos que sugieren una relación entre los niveles plasmáticos de las hormonas sexuales y la sintomatología de algunas enfermedades nerviosas, como la enfermedad de Parkinson $[63,64]$ o la epilepsia, donde se ha demostrado una correlación negativa ente los niveles de progesterona y el número de ataques epilépticos en las mujeres que sufren dicha enfermedad [65].

Por lo tanto, existen indicios de la relación entre los niveles de esteroides en sangre y el desarrollo de enfermedades nerviosas. La menopausia en las mujeres representa el caso más significativo de alteración en la concentración de esteroides en sangre. La disminución en la concentración de estradiol y progeste- 
rona plasmática tiene efectos negativos sobre el funcionamiento del sistema cardiovascular y sobre el componente óseo. En los últimos años, varios estudios han sugerido un posible efecto beneficioso de la terapia hormonal sustitutiva con estrógenos, sobre distintos aspectos cognitivos en las mujeres posmenopáusicas, que no sufren enfermedades neurológicas. Sin embargo, los estudios de observación que sugerían que el estradiol podría disminuir el riesgo y/o retrasar la aparición de enfermedades neurodegenerativas [66-68], no se han confirmado plenamente. De particular interés son los datos que hacen referencia a la enfermedad de Alzheimer: varios estudios han descrito que la terapia sustitutiva con estrógenos disminuye el riesgo de padecer dicha enfermedad [69-71]. Sin embargo, esta terapia no parece mejorar los síntomas de la enfermedad una vez que ésta se ha manifestado, e incluso puede empeorarlos. Algo parecido sucede con el infarto cerebral; aunque la mayoría de los estudios apuntan a que la terapia sustitutiva con estrógenos disminuye el riesgo de padecer un infarto cerebral [72], recientemente se ha descrito justo lo contrario [73]. Es difícil llegar a una conclusión en estos estudios, dado que muchos de ellos no son comparables entre sí. En particular, un factor que añade dificultad al análisis, es que la terapia hormonal aplicada no siempre es la misma. El efecto de la terapia hormonal con estrógenos tiene efectos bien diferentes si éstos se administran solos o en combinación con progesterona. También varía la formulación de progesterona utilizada. Por consiguiente, se necesitan más estudios y un mayor rigor en la agrupación de los mismos para alcanzar una mejor comprensión del fenómeno.

Recientemente, el National Institute of Health (NIH) ha recomendado la interrupción del uso de la terapia hormonal sustitutiva prolongada con estrógeno y progestina, ya que los resultados del estudio WHI (del inglés, Women's Health Initiative) sugieren que el uso prolongado durante varios años de esta terapia puede aumentar el riesgo de padecer cáncer de mama, cáncer de ovario e infarto. Este riesgo se considera superior a los efectos beneficiosos observados, como un aumento de la masa ósea, una disminución del riesgo de fracturas y una disminución del riesgo de cáncer colonorrectal. Los resultados del WHI también sugieren que la terapia prolongada con estrógeno y progestina aumenta el riesgo de Alzheimer y demencia vascular, aunque lo publicado hasta este momento se refiere a terapia hormonal en mujeres de 65 o más años y no en mujeres más jóvenes, para quienes los riesgos y beneficios cognitivos de esta terapia combinada no se conocen [74]. El estudio WHI ha recibido numerosas críticas, ya que el tratamiento se ha realizado sobre una población de mujeres de avanzada edad, muchos años después de la menopausia, cuando se considera que el tratamiento hormonal es más eficaz en el período perimenopáusico. Además, la progestina utilizada es acetato de medroxiprogesterona, un progestágeno sintético con efectos muy diferentes a la progesterona natural. Por otra parte, los resultados de un estudio paralelo del WHI con un tratamiento a largo plazo sólo con estrógeno, sin progesterona, también han demostrado efectos adversos.

En contraste con esta situación tan confusa sobre el efecto de las hormonas ováricas en el cerebro humano, el carácter beneficioso de los estrógenos en el $\mathrm{SN}$ es muy claro en los estudios realizados en animales. Se sabe, desde hace muchos años, que el estradiol es un factor que promueve la supervivencia y diferenciación de diferentes poblaciones neuronales en cultivo, como células hipotalámicas [75,76], células de la amígdala [77] o células hipocampales [78]. Además, se ha demostrado que el estradiol protege de la muerte neuronal inducida por multitud de agentes estresantes como los radicales libres [79], el péptido $\beta$-amiloide [80], y los aminoácidos excitadores glutamato [79, 81,82], AMPA [82], kainato [83] y NMDA [48,83].

Las cualidades neuroprotectoras del estradiol también se han puesto de manifiesto en experimentos in vivo. De esta manera, se ha demostrado que el estradiol reduce el daño isquémico producido por la oclusión de la arteria cerebral media en ratas [84-86], en ratones [87] y en gerbos [88]. Además, la hormona protege a las neuronas del sistema dopaminérgico negroestriatal de la toxicidad de MPTP (1-metil-4-fenil-1,2,3,6-tetrahidropiridina) [8991] o de la 6-hidroxidopamina [92] y bloquea la muerte por excitoxicidad mediada por kainato en el hilus del hipocampo [93]. Un dato interesante es que en la mayoría de los modelos experimentales, el estradiol tiene efectos neuroprotectores si se administra antes del estímulo lesivo, pero no si se administra una vez que la lesión ha tenido lugar. Este fenómeno concuerda con datos clínicos que muestran que la terapia sustitutiva con estrógenos no tiene efecto o incluso es perjudicial $[94,95]$ si se administra a sujetos con enfermedades neurológicas, como la de Alzheimer, ya diagnosticadas. Esto sugiere que los estrógenos actúan más como un factor preventivo que curativo en el desarrollo de enfermedades del SN [96,97].

El estradiol no es el único esteroide con proyección terapéutica en el tratamiento de las enfermedades del SN. La pregnenolona, la DHEA y la testosterona son esteroides precursores del estradiol y sus propiedades neuroprotectoras reciben cada vez más atención en la investigación. La pregnenolona es el primer esteroide sintetizado a partir del colesterol y también presenta cualidades neuroprotectoras; la pregnenolona protege a las células hipocampales de la línea HT-22 de la toxicidad del glutamato y de la proteína $\beta$-amiloide [98]. La testosterona también ha mostrado ser un agente protector eficaz en cultivos primarios de neuronas humanas [99]. La DHEA protege a las células hipocampales HT-22 de la toxicidad por glutamato [100], así como a cultivos primarios de neuronas hipocampales de la acción neurotóxica del AMPA y del ácido kaínico [101]. La administración de DHEA reduce también la muerte de las neuronas piramidales del hipocampo expuestas a la toxicidad del NMDA in vivo [101]. Además, la DHEA es neuroprotectora frente a las acciones neurotóxicas de la corticosterona [102], los radicales libres [57,103] y el MPTP [104,105], y reduce el volumen de infarto en la isquemia cerebral inducida por oclusión de la arteria cerebral media [106].

El hecho de que las concentraciones de DHEA experimenten una marcada disminución con la edad en el ser humano, unido a sus cada vez más ampliamente descritas propiedades neuroprotectoras, sugieren la posibilidad de que dicha hormona pueda ser una herramienta útil para el tratamiento de las disfunciones nerviosas asociadas a envejecimiento. Por otro lado, la sensación de bienestar y juventud descrita por sus consumidores y constatada en ensayos clínicos [107], sugiere su utilidad en el tratamiento de enfermedades relacionadas con el estado de ánimo. De hecho, existen un par de estudios que describen una mejoría en los síntomas de la depresión y de la distimia [108,109] con la administración de DHEA. Sin embargo, y aunque estos datos son prometedores, se necesita un mayor número de estudios clínicos que confirmen estas características terapéuticas. La mayoría de los datos que describen el efecto beneficioso de la DHEA sobre la función nerviosa provienen de estudios realizados en animales de experimentación, y la aplicación de los resultados al ser humano debe realizarse con cautela: la mayoría de ellos utilizan 
dosis suprafisiológicas del esteroide para conseguir el efecto y, lo que es más importante todavía, casi la totalidad de los estudios se han realizado en roedores, cuyas concentraciones fisiológicas de DHEA son casi indetectables en sangre. Los estudios deben, además, asegurar la inocuidad de un tratamiento a largo plazo, fenómeno este de gran importancia, dada la posibilidad de su transformación en esteroides potencialmente carcinogénicos, como el estradiol o la testosterona.

La pregnenolona, la DHEA y la testosterona pueden transformarse en estradiol a través de la acción de las enzimas esteroidogénicas. La aromatasa representa la última enzima que participa en dicha transformación. Los niveles de expresión de la enzima aromatasa son bajos en condiciones normales en el cerebro de ratas. Sin embargo, estos niveles experimentan un dramático aumento en los astrocitos cuando el tejido nervioso se somete a una lesión [59]. Esto sugiere que la síntesis de estradiol por acción de la aromatasa podría formar parte de la respuesta compensatoria del tejido nervioso a un daño. De hecho, se ha demostrado que la aromatasa cerebral es neuroprotectora [110] y que los efectos neuroprotectores de la pregnenolona, la DHEA y la testosterona dependen en parte de su conversión a estradiol por esta enzima [111].

La progesterona, y en particular sus metabolitos DHP y THP, también tienen propiedades neuroprotectoras. La THP tiene propiedades anticonvulsionantes, anestésicas, ansiolíticas y moduladoras del sueño en modelos experimentales animales [44]. La progesterona y sus metabolitos participan en el proceso de mielinización y constituyen uno de los factores secretados de manera autocrina por la célula de Schwann durante dicho proceso. Las células de Schwann, igual que los oligodendrocitos, son capaces de sintetizar progesterona [112-114], la cual estimula la formación de vainas de mielina en cultivos mixtos de neuronas y células de Schwann. Además, Chan et al pusieron de manifiesto en dichos cultivos mixtos, que había una inducción de enzimas esteroidogénicas que coincidía con el proceso de mielinización [115]. En particular, aumentaban los niveles de ARNm de la P450scc, responsable de la conversión de colesterol en pregnenolona y de la $3 \beta-H S D$, que convierte la pregnenolona en progesterona. El receptor de progesterona mostraba la misma tendencia que las enzimas esteroidogénicas. En otros experimentos se observó que la administración exógena de progesterona a los cultivos adelantaba el momento de inicio de la mielinización y duplicaba la tasa de formación de mielina [115].

Además de aumentar la incorporación de lípidos a la membrana de las células mielinizantes, la progesterona y sus derivados DHP y THP son capaces de modificar la expresión génica de las proteínas de la mielina P0 y PMP22. Melcangi et al han puesto de manifiesto que tanto progesterona como DHP modifican la expresión génica de $\mathrm{P} 0$. El otro metabolito de la progesterona, THP, a su vez, es capaz de influir también sobre los niveles de ARNm de PMP22 [116]. El papel de la progesterona en la mielinización no se restringe sólo al proceso en situaciones 'normales', sino que la progesterona y sus derivados también han mostrado ser agentes eficaces en la remielinización tras una lesión.
Koenig et al demostraron que la progesterona promovía la remielinización del nervio ciático del ratón después de dañarse por criolesión. La inhibición de la síntesis de progesterona -con trilostano, un inhibidor enzimático de la $3 \beta$-HSD - o de su receptor (con RU486), inhibe dicha remielinización. También se ha descrito que los niveles de ARNm de la $3 \beta$-HSD aumentan en el nervio ciático de rata después de una criolesión, y que este incremento es paralelo a los cambios en el ARNm para las proteínas de la mielina P0 y PMP22. Estos cambios, además, ocurren en el día 15 después de la lesión, un tiempo que se supone coincide con la formación de nuevos contactos entre la célula de Schwann y el axón en regeneración [117]. Un reciente estudio ha demostrado el efecto beneficioso de la progesterona y sus derivados DHP y THP en la prevención del deterioro del nervio periférico con el envejecimiento en ratas [118]. Otro reciente estudio ha demostrado la eficacia terapéutica de un antagonista del receptor de progesterona en un modelo animal de la enfermedad de Charcot-MarieTooth [119]. Todos estos datos señalan a la progesterona y a sus derivados y al receptor de progesterona como herramientas terapéuticas potenciales para el tratamiento de situaciones en las que haya una pérdida y/o una alteración en la mielina.

\section{CONCLUSIONES: ALTERNATIVAS TERAPÉUTICAS AL TRATAMIENTO HORMONAL}

Dada la limitación que plantea el uso sistémico de hormonas como terapia neuroprotectora por sus consecuencias endocrinas y por los riesgos antes mencionados, se necesitan encontrar estrategias alternativas para un posible tratamiento neuroprotector, aprovechando las propiedades neuroprotectoras de los esteroides. Existen varias posibilidades que merecen estudiarse experimentalmente. Teniendo en cuenta la capacidad de esteroidogénesis del tejido nervioso, una alternativa a la administración exógena de esteroides neuroprotectores sería la de aumentar localmente su síntesis en el cerebro, lo cual evitaría efectos secundarios no deseados debidos a su acción periférica. En este sentido, las proteínas StAR y PBR o las enzimas implicadas en la esteroidogénesis pueden ser buenos candidatos de estudio. Por ejemplo, una manera de aumentar la síntesis de esteroides sería utilizar agonistas de PBR. De hecho, se ha descrito que la inyección de un agonista de PBR en el hipocampo aumenta la síntesis local de THP y este aumento se asocia a efectos ansiolíticos [34]. Otra molécula importante es la aromatasa, la enzima responsable de la formación de estradiol. El gen humano de la aromatasa tiene siete promotores conocidos cuyo uso varía según el tejido y la situación funcional [120]. Por lo tanto, es teóricamente posible el diseño de tratamientos farmacológicos específicos que induzcan la aromatasa en el SN y no en otros tejidos. Otras estrategias neuroprotectoras tienen que ver con los mecanismos de neuroprotección mediados por los receptores nucleares de hormonas esteroides. Actualmente se desarrollan nuevos moduladores selectivos de estos receptores y es posible que se puedan conseguir moléculas con capacidad neuroprotectora y sin efectos periféricos no deseados.

\section{BIBLIOGRAFÍA}

1. Morrow AL, Suzdak PD, Paul SM. Steroid hormone metabolites potentiate GABA receptor-mediated chloride ion flux with nanomolar potency. Eur J Pharmacol 1987; 142: 483-5.

2. Bitran D, Hilvers RJ, Kellogg CK. Anxiolytic effects of 3 alpha-hydroxy5 alpha[beta]-pregnan-20-one: endogenous metabolites of progesterone that are active at the $\mathrm{GABA}_{\mathrm{A}}$ receptor. Brain Res 1991; 561: 157-61.
3. Uzunov DP, Cooper TB, Costa E, Guidotti A. Fluoxetine-elicited changes in brain neurosteroid content measured by negative ion mass fragmentography. Proc Natl Acad Sci USA 1996; 93: 12599-604.

4. Nechmad A, Maayan R, Spivak B, Ramadan E, Poyurovsky M, Weizman A. Brain neurosteroid changes after paroxetine administration in mice. Eur Neuropsychopharmacol 2003; 13: 327-32. 
5. Corpechot C, Robel P, Axelson M, Sjovall J, Baulieu EE. Characterization and measurement of dehydroepiandrosterone sulfate in rat brain. Proc Natl Acad Sci USA 1981; 78: 4704-7.

6. Corpechot C, Synguelakis M, Talha S, Axelson M, Sjovall J, Vihko R, et al. Pregnenolone and its sulfate ester in the rat brain. Brain Res 1983; 270: 119-25.

7. Zhao HF, Labrie C, Simard J, De Launoit Y, Trudel C, Martel C, et al. Characterization of rat 3 beta-hydroxysteroid dehydrogenase/delta 5delta 4 isomerase cDNAs and differential tissue-specific expression of the corresponding mRNAs in steroidogenic and peripheral tissues. J Biol Chem 1991; 266: 583-93.

8. Mellon SH, Deschepper CF. Neurosteroid biosynthesis: genes for adrenal steroidogenic enzymes are expressed in the brain. Brain Res 1993; 629: 283-92.

9. Sanne JL, Krueger KE. Expression of cytochrome P450 side-chain cleavage enzyme and 3 beta-hydroxysteroid dehydrogenase in the rat central nervous system: a study by polymerase chain reaction and in situ hybridization. J Neurochem 1995; 65: 528-36.

10. Le Goascogne C, Robel P, Gouezou M, Sananes N, Baulieu EE, Waterman M. Neurosteroids: cytochrome P-450scc in rat brain. Science 1987; 237: 1212-5

11. Jung-Testas I, Hu ZY, Baulieu EE, Robel P. Neurosteroids: biosynthesis of pregnenolone and progesterone in primary cultures of rat glial cells. Endocrinology 1989; 125: 2083-91.

12. Zwain IH, Yen SS. Neurosteroidogenesis in astrocytes, oligodendrocytes, and neurons of cerebral cortex of rat brain. Endocrinology 1999; 140: $3843-52$.

13. Tsutsui K, Ukena K, Usui M, Sakamoto H, Takase M. Novel brain function: biosynthesis and actions of neurosteroids in neurons. Neurosci Res 2000; 36: 261-73.

14. West LA, Horvat RD, Roess DA, Barisas BG, Juengel JL, Niswender GD. Steroidogenic acute regulatory protein and peripheral-type benzodiazepine receptor associate at the mitochondrial membrane. Endocrinology 2001; 142: 502-5.

15. Mathieu AP, Fleury A, Ducharme L, Lavigne P, LeHoux JG. Insights into steroidogenic acute regulatory protein (StAR)-dependent cholesterol transfer in mitochondria: evidence from molecular modeling and structure-based thermodynamics supporting the existence of partially unfolded states of StAR. J Mol Endocrinol 2002; 29: 327-45.

16. Sierra A, Lavaque E, Pérez-Martín M, Azcoitia I, Hales DB, GarcíaSegura LM. Steroidogenic acute regulatory protein in the rat brain: cellular distribution, developmental regulation and overexpression after injury. Eur J Neurosci 2003; 18: 1458-67.

17. Benmessahel Y, Guennoun R, Cadepond F, Baulieu EE, Schumacher M, Groyer G. Expression of steroidogenic acute regulatory protein in cultured Schwann cells and its regulation by cAMP. Ann NY Acad Sci 2002; 973: 83-7.

18. Lacor P, Gandolfo P, Tonon MC, Brault E, Dalibert I, Schumacher M, et al. Regulation of the expression of peripheral benzodiazepine receptors and their endogenous ligands during rat sciatic nerve degeneration and regeneration: a role for PBR in neurosteroidogenesis. Brain Res 1999; 815: 70-80.

19. Lacor P, Benavides J, Ferzaz B. Enhanced expression of the peripheral benzodiazepine receptor (PBR) and its endogenous ligand octadecaneuropeptide (ODN) in the regenerating adult rat sciatic nerve. Neurosci Lett 1996; 220: 61-5.

20. Cagnin A, Myers R, Gunn RN, Lawrence AD, Stevens T, Kreutzberg $\mathrm{GW}$, et al. In vivo visualization of activated glia by [11C]-K11195PET following herpes encephalitis reveals projected neuronal damage beyond the primary focal lesion. Brain 2001; 124: 2014-27.

21. Agnello D, Carvelli L, Muzio V, Villa P, Bottazzi B, et al. Increased peripheral benzodiazepine binding sites and pentraxin 3 expression in the spinal cord during EAE: relation to inflammatory cytokines and modulation by dexamethasone and rolipram. J Neuroimmunol 2000; 109: 105-11.

22. Debruyne JC, Versijpt J, Van Laere KJ, De Vos F, Keppens J, Strijckmans K, et al. PET visualization of microglia in multiple sclerosis patients using [11C]PK11195. Eur J Neurol 2003; 10: 257-64

23. Stephenson DT, Schober DA, Smalstig EB, Mincy RE, Gehlert DR, Clemens JA. Peripheral benzodiazepine receptors are colocalized with activated microglia following transient global forebrain ischemia in the rat. J Neurosci 1995; 15: 5263-74.

24. Raghavendra RV, Dogan A, Bowen KK, Dempsey RJ. Traumatic brain injury leads to increased expression of peripheral-type benzodiazepine receptors, neuronal death, and activation of astrocytes and microglia in rat thalamus. Exp Neurol 2000; 161: 102-14.

25. Altar CA, Baudry M. Systemic injection of kainic acid: gliosis in olfactory and limbic brain regions quantified with [3H]PK 11195 binding autoradiography. Exp Neurol 1990; 109: 333-41.
26. Kuhlmann AC, Guilarte TR. Cellular and subcellular localization of peripheral benzodiazepine receptors after trimethyltin neurotoxicity. J Neurochem 2000; 74: 1694-704.

27. Kuhlmann AC, Guilarte TR. The peripheral benzodiazepine receptor is a sensitive indicator of domoic acid neurotoxicity. Brain Res 1997; 751: $281-8$

28. Vowinckel E, Reutens D, Becher B, Verge G, Evans A, Owens T, et al. PK11195 binding to the peripheral benzodiazepine receptor as a marker of microglia activation in multiple sclerosis and experimental autoimmune encephalomyelitis. J Neurosci Res 1997; 50: 345-53.

29. Banati RB, Newcombe J, Gunn RN, Cagnin A, Turkheimer F, Heppner $\mathrm{F}$, et al. The peripheral benzodiazepine binding site in the brain in multiple sclerosis: quantitative in vivo imaging of microglia as a measure of disease activity. Brain 2000; 123: 2321-37.

30. Banati RB. Visualising microglial activation in vivo. Glia 2002; 40: 206-17.

31. Xia W, Spector S, Hardy L, Zhao S, Saluk A, Alemane L, et al. Tumor selective G2/M cell cycle arrest and apoptosis of epithelial and hematological malignancies by BBL22, a benzazepine. Proc Natl Acad Sci USA 2000; 97: 7494-9.

32. Sutter AP, Maaser K, Hopfner M, Barthel B, Grabowski P, Faiss S, et al. Specific ligands of the peripheral benzodiazepine receptor induce apoptosis and cell cycle arrest in human esophageal cancer cells. Int $\mathbf{J}$ Cancer 2002; 102: 318-27.

33. Serra M, Madau P, Chessa MF, Caddeo M, Sanna E, Trapani G, et al. 2-Phenyl-imidazo[1,2-a]pyridine derivatives as ligands for peripheral benzodiazepine receptors: stimulation of neurosteroid synthesis and anticonflict action in rats. Br J Pharmacol 1999; 127: 177-87.

34. Bitran D, Foley M, Audette D, Leslie N, Frye CA. Activation of peripheral mitochondrial benzodiazepine receptors in the hippocampus stimulates allopregnanolone synthesis and produces anxiolytic-like effects in the rat. Psychopharmacology (Berl) 2000; 151: 64-71.

35. Nakamura K, Fukunishi I, Nakamoto Y, Iwahashi K, Yoshii M. Peripheral-type benzodiazepine receptors on platelets are correlated with the degrees of anxiety in normal human subjects. Psychopharmacology (Berl) 2002; 162: 301-3.

36. Kelly MJ, Levin ER. Rapid actions of plasma membrane estrogen receptors. Trends Endocrinol Metab 2001; 12: 152-6.

37. Simoncini T, Genazzani AR. Non-genomic actions of sex steroid hormones. Eur J Endocrinol 2003; 148: 281-92.

38. Rossato M, Nogara A, Merico M, Ferlin A, Foresta C. Identification of functional binding sites for progesterone in rat Leydig cell plasma membrane. Steroids 1999; 64: 168-75.

39. Nemere I, Farach-Carson MC. Membrane receptors for steroid hormones: a case for specific cell surface binding sites for vitamin D metabolites and estrogens. Biochem Biophys Res Commun 1998; 248: 443-9.

40. Benten WP, Lieberherr M, Giese G, Wrehlke C, Stamm O, Sekeris CE, et al. Functional testosterone receptors in plasma membranes of $\mathrm{T}$ cells. FASEB J 1999; 13: 123-33.

41. Liu D, Dillon JS. Dehydroepiandrosterone activates endothelial cell nitric-oxide synthase by a specific plasma membrane receptor coupled to Galpha(i2,3). J Biol Chem 2002; 277: 21379-88.

42. Fiorelli G, Gori F, Frediani U, Franceschelli F, Tanini A, Tosti-Guerra $\mathrm{C}$, et al. Membrane binding sites and non-genomic effects of estrogen in cultured human pre-osteoclastic cells. J Steroid Biochem Mol Biol 1996; 59: 233-40.

43. Rupprecht R, Holsboer F. Neuroactive steroids: mechanisms of action and neuropsychopharmacological perspectives. Trends Neurosci 1999; 22: 410-6.

44. Rupprecht R. Neuroactive steroids: mechanisms of action and neuropsychopharmacological properties. Psychoneuroendocrinology 2003; 28: 139-68.

45. Majewska MD, Harrison NL, Schwartz RD, Barker JL, Paul SM. Steroid hormone metabolites are barbiturate-like modulators of the GABA receptor. Science 1986; 232: 1004-7.

46. Biegon A, McEwen BS. Modulation by estradiol of serotonin receptors in brain. J Neurosci 1982; 2: 199-205.

47. Wetzel CH, Hermann B, Behl C, Pestel E, Rammes G, Zieglgansberger W, et al. Functional antagonism of gonadal steroids at the 5-hydroxytryptamine type 3 receptor. Mol Endocrinol 1998; 12: 1441-51.

48. Weaver CE Jr, Park-Chung M, Gibbs TT, Farb DH. 17beta-Estradiol protects against NMDA-induced excitotoxicity by direct inhibition of NMDA receptors. Brain Res 1997; 761: 338-41.

49. Park-Chung M, Wu FS, Farb DH. 3 alpha-hydroxy-5 beta-pregnan-20one sulfate: a negative modulator of the NMDA-induced current in cultured neurons. Mol Pharmacol 1994; 46: 146-50.

50. Wu FS, Gibbs TT, Farb DH. Pregnenolone sulfate: a positive allosteric modulator at the N-methyl-D-aspartate receptor. Mol Pharmacol 1991; 40: 333-6. 
51. Wu FS, Gibbs TT, Farb DH. Inverse modulation of gamma-aminobutyric acid- and glycine-induced currents by progesterone. Mol Pharmacol 1990; 37: 597-602.

52. Valera S, Ballivet M, Bertrand D. Progesterone modulates a neuronal nicotinic acetylcholine receptor. Proc Natl Acad Sci USA 1992; 89: 9949-53

53. Bullock AE, Clark AL, Grady SR, Robinson SF, Slobe BS, Marks MJ, et al. Neurosteroids modulate nicotinic receptor function in mouse striatal and thalamic synaptosomes. J Neurochem 1997; 68: 2412-23.

54. Rubin LL, Gatchalian CL, Rimon G, Brooks SF. The molecular mechanisms of neuronal apoptosis. Curr Opin Neurobiol 1994; 4: 696-702.

55. Behl C. Estrogen can protect neurons: modes of action. J Steroid Biochem Mol Biol 2002; 83: 195-7.

56. Morin C, Zini R, Simon N, Tillement JP. Dehydroepiandrosterone and alpha-estradiol limit the functional alterations of rat brain mitochondria submitted to different experimental stresses. Neuroscience 2002; 115: 415-24

57. Bastianetto S, Ramassamy C, Poirier J, Quirion R. Dehydroepiandrosterone (DHEA) protects hippocampal cells from oxidative stressinduced damage. Brain Res Mol Brain Res 1999; 66: 35-41.

58. Shin CY, Choi JW, Jang ES, Ju C, Kim WK, Kim HC, et al. Dehydroepiandrosterone inhibits the death of immunostimulated rat C6 glioma cells deprived of glucose. Brain Res 2001; 922: 267-75.

59. García-Segura LM, Wozniak A, Azcoitia I, Rodríguez JR, Hutchison RE, Hutchison JB. Aromatase expression by astrocytes after brain injury: implications for local estrogen formation in brain repair. Neuroscience 1999; 89: 567-78.

60. Stein DG. Brain damage, sex hormones and recovery: a new role for progesterone and estrogen? Trends Neurosci 2001; 24: 386-91.

61. Azcoitia I, Fernández-Galaz C, Sierra A, Garcóa-Segura LM. Gonadal hormones affect neuronal vulnerability to excitotoxin-induced degeneration. J Neurocytol 1999; 28: 699-710.

62. McCullough LD, Hurn PD. Estrogen and ischemic neuroprotection: an integrated view. Trends Endocrinol Metab 2003; 14: 228-35.

63. Kompoliti K, Comella CL, Jaglin JA, Leurgans S, Raman R, Goetz $\mathrm{CG}$. Menstrual-related changes in motoric function in women with Parkinson's disease. Neurology 2000; 55: 1572-5.

64. Saunders-Pullman R. Estrogens and Parkinson disease: neuroprotective, symptomatic, neither, or both? Endocrine 2003; 21: 81-7.

65. Backstrom T. Epileptic seizures in women related to plasma estrogen and progesterone during the menstrual cycle. Acta Neurol Scand 1976; 54: 321-47.

66. Paganini-Hill A, Henderson VW. Estrogen replacement therapy and risk of Alzheimer disease. Arch Intern Med 1996; 156: 2213-7.

67. Kawas C, Resnick S, Morrison A, Brookmeyer R, Corrada M, Zonderman A, et al. A prospective study of estrogen replacement therapy and the risk of developing Alzheimer's disease: the Baltimore Longitudinal Study of Aging. Neurology 1997; 48: 1517-21.

68. Wise PM, Dubal DB, Wilson ME, Rau SW, Liu Y. Estrogens: trophic and protective factors in the adult brain. Front Neuroendocrinol 2001; 22: 33-66.

69. Tang MX, Jacobs D, Stern Y, Marder K, Schofield P, Gurland B, et al. Effect of oestrogen during menopause on risk and age at onset of Alzheimer's disease. Lancet 1996; 348: 429-32.

70. Yaffe K, Sawaya G, Lieberburg I, Grady D. Estrogen therapy in postmenopausal women: effects on cognitive function and dementia. JAMA 1998; 279: 688-95.

71. Costa MM, Reus VI, Wolkowitz OM, Manfredi F, Lieberman M. Estrogen replacement therapy and cognitive decline in memory-impaired post-menopausal women. Biol Psychiatry 1999; 46: 182-8.

72. Paganini-Hill A. Estrogen replacement therapy and stroke. Prog Cardiovasc Dis 1995; 38: 223-42.

73. Rossouw JE, Anderson GL, Prentice RL, LaCroix AZ, Kooperberg C, Stefanick ML, et al. Risks and benefits of estrogen plus progestin in healthy postmenopausal women: principal results From the Women's Health Initiative randomized controlled trial. JAMA 2002; 288: 321-33.

74. Shumaker SA, Legault C, Rapp SR, Thal L, Wallace RB, Ockene JK, et al. Estrogen plus progestin and the incidence of dementia and mild cognitive impairment in postmenopausal women: the Women's Health Initiative Memory Study: a randomized controlled trial. JAMA 2003; 289: 2651-62.

75. Chowen JA, Torres-Aleman I, García-Segura LM. Trophic effects of estradiol on fetal rat hypothalamic neurons. Neuroendocrinology 1992 . 56: 895-901

76. Duenas M, Torres-Alemán I, Naftolin F, García-Segura LM. Interaction of insulin-like growth factor-I and estradiol signalling pathways on hypothalamic neuronal differentiation. Neuroscience 1996; 74: 531-9.

77. Arimatsu Y, Hatanaka H. Estrogen treatment enhances survival of cul- tured fetal rat amygdala neurons in a defined medium. Brain Res 1986; 391: 151-9.

78. Sudo S, Wen TC, Desaki J, Matsuda S, Tanaka J, Arai T, et al. Betaestradiol protects hippocampal CA1 neurons against transient forebrain ischemia in gerbil. Neurosci Res 1997; 29: 345-54.

79. Sawada H, Ibi M, Kihara T, Urushitani M, Akaike A, Shimohama S. Estradiol protects mesencephalic dopaminergic neurons from oxidative stress-induced neuronal death. J Neurosci Res 1998; 54: 707-19.

80. Bonnefont AB, Munoz FJ, Inestrosa NC. Estrogen protects neuronal cells from the cytotoxicity induced by acetylcholinesterase-amyloid complexes. FEBS Lett 1998; 441: 220-4.

81. Singer CA, Rogers KL, Strickland TM, Dorsa DM. Estrogen protects primary cortical neurons from glutamate toxicity. Neurosci Lett 1996; 212: 13-6.

82. Zaulyanov LL, Green PS, Simpkins JW. Glutamate receptor requirement for neuronal death from anoxia-reoxygenation: an in Vitro model for assessment of the neuroprotective effects of estrogens. Cell Mol Neurobiol 1999; 19: 705-18.

83. Regan RF, Guo Y. Estrogens attenuate neuronal injury due to hemoglobin, chemical hypoxia, and excitatory amino acids in murine cortical cultures. Brain Res 1997; 764: 133-40.

84. Simpkins JW, Rajakumar G, Zhang YQ, Simpkins CE, Greenwald D, $\mathrm{Yu}$ CJ, et al. Estrogens may reduce mortality and ischemic damage caused by middle cerebral artery occlusion in the female rat. J Neurosurg 1997; 87: 724-30.

85. Dubal DB, Kashon ML, Pettigrew LC, Ren JM, Finklestein SP, Rau $\mathrm{SW}$, et al. Estradiol protects against ischemic injury. J Cereb Blood Flow Metab 1998; 18: 1253-8.

86. Wang Q, Santizo R, Baughman VL, Pelligrino DA, Iadecola C. Estrogen provides neuroprotection in transient forebrain ischemia through perfusion-independent mechanisms in rats. Stroke 1999; 30: 630-7.

87. Culmsee C, Vedder H, Ravati A, Junker V, Otto D, Ahlemeyer B, et al. Neuroprotection by estrogens in a mouse model of focal cerebral ischemia and in cultured neurons: evidence for a receptor-independent antioxidative mechanism. J Cereb Blood Flow Metab 1999; 19: 1263-9.

88. Chen J, Adachi N, Liu K, Arai T. The effects of 17beta-estradiol on ischemia-induced neuronal damage in the gerbil hippocampus. Neuroscience 1998; 87: 817-22.

89. Dluzen DE, McDermott JL, Liu B. Estrogen alters MPTP-induced neurotoxicity in female mice: effects on striatal dopamine concentrations and release. J Neurochem 1996; 66: 658-66.

90. Dluzen DE, McDermott JL, Liu B. Estrogen as a neuroprotectant against MPTP-induced neurotoxicity in C57/B1 mice. Neurotoxicol Teratol 1996; 18: 603-6.

91. Callier S, Morissette M, Grandbois M, Di Paolo T. Stereospecific prevention by 17beta-estradiol of MPTP-induced dopamine depletion in mice. Synapse 2000; 37: 245-51.

92. Dluzen D. Estrogen decreases corpus striatal neurotoxicity in response to 6-hydroxydopamine. Brain Res 1997; 767: 340-4.

93. Azcoitia I, Sierra A, García-Segura LM. Estradiol prevents kainic acidinduced neuronal loss in the rat dentate gyrus. Neuroreport 1998; 9 : 3075-9.

94. Henderson VW, Paganini-Hill A, Miller BL, Elble RJ, Reyes PF, Shoupe $\mathrm{D}$, at al. Estrogen for Alzheimer's disease in women: randomized, double-blind, placebo-controlled trial. Neurology 2000; 54: 295-301.

95. Mulnard RA, Cotman CW, Kawas C, Van Dyck CH, Sano M, Doody $\mathrm{R}$, et al. Estrogen replacement therapy for treatment of mild to moderate Alzheimer disease: a randomized controlled trial. Alzheimer's Disease Cooperative Study. JAMA 2000; 283: 1007-15.

96. García-Segura LM, Azcoitia I, Don Carlos LL. Neuroprotection by estradiol. Prog Neurobiol 2001; 63: 29-60.

97. Wise PM. Estrogens: protective or risk factors in brain function? Prog Neurobiol 2003; 69: 181-91.

98. Gursoy E, Cardounel A, Kalimi M. Pregnenolone protects mouse hippocampal (HT-22) cells against glutamate and amyloid beta protein toxicity. Neurochem Res 2001; 26: 15-21.

99. Hammond J, Le Q, Goodyer C, Gelfand M, Trifiro M, LeBlanc A. Testosterone-mediated neuroprotection through the androgen receptor in human primary neurons. J Neurochem 2001; 77: 1319-26.

100. Cardounel A, Regelson W, Kalimi M. Dehydroepiandrosterone protects hippocampal neurons against neurotoxin- induced cell death: mechanism of action. Proc Soc Exp Biol Med 1999; 222: 145-9.

101. Kimonides VG, Khatibi NH, Svendsen CN, Sofroniew MV, Herbert J. Dehydroepiandrosterone (DHEA) and DHEA-sulfate (DHEAS) protect hippocampal neurons against excitatory amino acid-induced neurotoxicity. Proc Natl Acad Sci USA 1998; 95: 1852-7.

102. Kimonides VG, Spillantini MG, Sofroniew MV, Fawcett JW, Herbert J Dehydroepiandrosterone antagonizes the neurotoxic effects of corti- 
costerone and translocation of stress-activated protein kinase 3 in hippocampal primary cultures. Neuroscience 1999; 89: 429-36.

103. Aragno M, Parola S, Brignardello E, Mauro A, Tamagno E, Manti R, et al. Dehydroepiandrosterone prevents oxidative injury induced by transient ischemia/reperfusion in the brain of diabetic rats. Diabetes 2000; 49: $1924-31$

104. Tomas-Camardiel M, Sánchez-Hidalgo MC, Sánchez del Pino MJ, Navarro A, Machado A, Cano J. Comparative study of the neuroprotective effect of dehydroepiandrosterone and 17beta-estradiol against 1methyl-4-phenylpyridium toxicity on rat striatum. Neuroscience 2002; 109: 569-84.

105. D'Astous M, Morissette M, Tanguay B, Callier S, Di Paolo T. Dehydroepiandrosterone (DHEA) such as 17beta-estradiol prevents MPTPinduced dopamine depletion in mice. Synapse 2003; 47: 10-4.

106. Li H, Klein G, Sun P, Buchan AM. Dehydroepiandrosterone (DHEA) reduces neuronal injury in a rat model of global cerebral ischemia. Brain Res 2001; 888: 263-6.

107. Morales AJ, Haubrich RH, Hwang JY, Asakura H, Yen SS. The effect of six months treatment with a $100 \mathrm{mg}$ daily dose of dehydroepiandrosterone (DHEA) on circulating sex steroids, body composition and muscle strength in age-advanced men and women. Clin Endocrinol (Oxf) 1998; 49: 421-32.

108. Wolkowitz OM, Reus VI, Keebler A, Nelson N, Friedland M, Brizendine $\mathrm{L}$, et al. Double-blind treatment of major depression with dehydroepiandrosterone. Am J Psychiatry 1999; 156: 646-9.

109. Bloch M, Schmidt PJ, Danaceau MA, Adams LF, Rubinow DR. Dehydroepiandrosterone treatment of midlife dysthymia. Biol Psychiatry 1999; 45: 1533-41

110. Azcoitia I, Sierra A, Veiga S, García-Segura LM. Aromatase expression by reactive astroglia is neuroprotective. Ann NY Acad Sci 2003; 1007: 298-305

111. Veiga S, García-Segura LM, Azcoitia I. Neuroprotection by the ste-

\section{PROPIEDADES NEUROPROTECTORAS DE LOS \\ ESTEROIDES SEXUALES Y LOS NEUROESTEROIDES}

Resumen. Introducción. El sistema nervioso es una diana de las hormonas esteroides y también un tejido esteroidogénico, y produce esteroides que actúan de una forma paracrina o autocrina sobre neuronas y glía. Los esteroides formados en el tejido nervioso se denominan neuroesteroides, para diferenciarlos, por su origen, de los esteroides periféricos, aunque tienen la misma estructura molecular que éstos. Desarrollo. Se analiza la capacidad de las neuronas y las células de glía de sintetizar esteroides, y se describe el papel de algunas moléculas clave en la esteroidogénesis, como la proteína de regulación aguda de esteroidogénesis, el receptor periférico de benzodiacepinas y la enzima aromatasa, que cataliza la conversión de testosterona en estradiol. También se describen los diversos mecanismos de acción de los esteroides hormonales y los neuroesteroides en el sistema nervioso. Éstos incluyen, tanto la regulación de la síntesis de proteínas por neuronas y glía, mediante acciones sobre receptores nucleares, como efectos rápidos mediados por receptores de membrana o la modulación alostérica de receptores para neurotransmisores. Se revisan las evidencias clínicas y experimentales de los efectos neuroprotectores de los esteroides sexuales y neuroesteroides y las limitaciones de la terapia hormonal sustitutiva, tras la menopausia. Conclusiones. Dada la limitación que plantea el uso sistémico de hormonas como terapia neuroprotectora, se necesitan encontrar estrategias alternativas que aprovechen las propiedades neuroprotectoras de los esteroides, tales como aumentar localmente su síntesis en el cerebro o desarrollar moléculas que activen a los receptores de esteroides en el sistema nervioso y no en los órganos periféricos. [REV NEUROL 2004; 39: 1043-51]

Palabras clave. Aromatasa. Dehidroepiandrosterona. Esteroidogénesis. Estradiol. Neuroesteroides. Neuroprotección. Pregnenolona. Progesterona. Proteína de regulación aguda de esteroidogénesis. Receptor periférico de benzodiacepinas. Testosterona. roids pregnenolone and dehydroepiandrosterone is mediated by the enzyme aromatase. J Neurobiol 2003; 56: 398-406.

112. Koenig HL, Schumacher M, Ferzaz B, Thi AN, Ressouches A, Guennoun R, et al. Progesterone synthesis and myelin formation by Schwann cells. Science 1995; 268: 1500-3.

113. Baulieu E, Schumacher M. Progesterone as a neuroactive neurosteroid, with special reference to the effect of progesterone on myelination. Steroids 2000; 65: 605-12.

114. Gago N, Akwa Y, Sananes N, Guennoun R, Baulieu EE, El Etr M, et al. Progesterone and the oligodendroglial lineage: stage-dependent biosynthesis and metabolism. Glia 2001; 36: 295-308.

115. Chan JR, Phillips LJ, Glaser M. Glucocorticoids and progestins signal the initiation and enhance the rate of myelin formation. Proc Natl Acad Sci USA 1998; 95: 10459-64.

116. Melcangi RC, Magnaghi V, Cavarretta I, Zucchi I, Bovolin P, D’Urso $\mathrm{D}$, et al. Progesterone derivatives are able to influence peripheral myelin protein 22 and P0 gene expression: possible mechanisms of action. J Neurosci Res 1999; 56: 349-57.

117. Robert F, Guennoun R, Desarnaud F, Do-Thi A, Benmessahel Y, Baulieu EE, et al. Synthesis of progesterone in Schwann cells: regulation by sensory neurons. Eur J Neurosci 2001; 13: 916-24.

118. Azcoitia I, Leonelli E, Magnaghi V, Veiga S, García-Segura LM, Melcangi RC. Progesterone and its derivatives dihydroprogesterone and tetrahydroprogesterone reduce myelin fiber morphological abnormalities and myelin fiber loss in the sciatic nerve of aged rats. Neurobiol Aging 2003; 24: 853-60.

119. Sereda MW, Meyer zu HG, Suter U, Uzma N, Nave KA. Therapeutic administration of progesterone antagonist in a model of CharcotMarie-Tooth disease (CMT-1A). Nat Med 2003; 9: 1533-7.

120. Kamat A, Hinshelwood MM, Murry BA, Mendelson CR. Mechanisms in tissue-specific regulation of estrogen biosynthesis in humans. Trends Endocrinol Metab 2002; 13: 122-8.

\section{PROPRIEDADES NEUROPROTECTORAS DOS}

\section{ESTERÓIDES SEXUAIS E OS NEUROESTERÓIDES}

Resumo. Introdução. O sistema nervoso é um alvo das hormonas esteróides e também um tecido esteroidogénico, e produz esteróides que actuam de uma forma parácrina ou autócrina sobre os neurónios e a glia. Os esteróides formados no tecido nervoso denominam-se neuroesteróides, para diferenciá-los, pela sua origem, dos esteróides periféricos, embora possuam a mesma estrutura molecular que estes. Desenvolvimento. Analisa-se a capacidade dos neurónios e das células da glia sintetizarem esteróides, e descreve-se o papel de algumas moléculas-chave na esteroidogénese, como a proteína de regulação aguda da esteroidogénese, o receptor periférico das benzodiazepinas e a enzima aromatase, que cataliza a conversão da testosterona em estradiol. Também se descrevem os diversos mecanismos de acção dos esteróides hormonais e os neuroesteróides hormonais e os neuroesteróides no sistema nervoso. Estes incluem tanto a regulação da síntese de proteínas por neurónios e glia, mediante acções sobre receptores nucleares, como efeitos rápidos mediados por receptores de membrana ou a modulação alostérica dos receptores para neurotransmissores. Revêem-se as evidências clínicas e experimentais dos efeitos neuroprotectores dos esteróides sexuais e neuroesteróides e as limitações da terapia hormonal de substituição, após a menopausa. Conclusões. Dada a limitação que estabelece o uso sistémico de hormonas como terapia neuroprotectora, é necessário encontrar estratégias alternativas que aproveitem as propriedades neuroprotectoras dos esteróides, tais como aumentar localmente a sua síntese no cérebro ou desenvolver moléculas que activem os receptores de esteróides no sistema nervoso e não nos órgãos periféricos. [REV NEUROL 2004; 39: 1043-51]

Palavras chave. Aromatase. Dehidroepiandrosterona. Esteroidogénese. Estradiol. Neuroesteróides. Neuroprotecção. Pregnenolona. Progesterona. Proteína de regulação aguda de esteroidogénese. Receptor periférico das benzodiazepinas. Testosterona. 